\title{
The Effect of Training Nursery Staff in Breastfeeding Guidance on the Duration of Breastfeeding in Healthy Term Infants
}

\author{
E.S. SHINWELL, ${ }^{1}$ Y. CHURGIN, ${ }^{1}$ M. SHLOMO, ${ }^{1}$ M. SHANI, ${ }^{2}$ and O. FLIDEL-RIMON ${ }^{1}$
}

\begin{abstract}
Background: Promoting breastfeeding is a central aim of child health care. It is critical to develop approaches that are inexpensive, effective, and suitable across cultural and socioeconomic groups.

Objective: To study the effect of training perinatal-neonatal nursing and medical staff in breastfeeding guidance on the duration of breastfeeding in a middle-income urban population.

Methods: This was an interventional study with data collection before and after. The intervention was an intensive course on breastfeeding guidance provided to all of the neonatal nurses and midwives in a local general hospital (2001-2002). Data were collected on two cohorts of mothers and infants (before $-1999[n=471]$, after $-2003[n=364]$ ) regarding the duration of breastfeeding and factors influencing its discontinuation.

Results: The rate of breastfeeding initiation rose from $84 \%$ to $93 \%(p=0.0001)$ and the mean duration of breastfeeding rose from $3.7 \pm 3.7$ to $5.6 \pm 4.3$ months $(p=0.0001)$. The rate of breastfeeding in the delivery room rose from $3 \%$ to $37 \%(p=0.0001)$. Satisfaction with breastfeeding guidance in the hospital rose from $43 \%$ to $79 \%(p=0.0001)$. However, there was no change in the proportion of mothers who planned to breastfeed this infant $(88 \%$ in both cohorts) and no significant differences in the reasons given by the mothers for stopping breastfeeding.

Conclusion: Training hospital nursery staff in breastfeeding guidance is a potential, costeffective intervention even in settings with relatively high rates of breastfeeding.
\end{abstract}

\section{INTRODUCTION}

$\mathbf{P}$ ROMOTING BREASTFEEDING is a central aim of child health care. Indeed, the challenge of achieving real and lasting improvement in rates and duration of breastfeeding is so daunting that the World Health Organization has encouraged member states to "strengthen activities and develop new approaches" toward this aim. ${ }^{1}$ Such efforts may range from national legislation, through health service and hospital policies to individual consultations.

Studies of programs of breastfeeding education for pregnant women and nursing mothers have proven efficacy. ${ }^{2}$ However, such interventions may be effective only as long as the program is available. By comparison, intensive training of the health professionals who pro-

\footnotetext{
${ }^{1}$ Department of Neonatology, Kaplan Medical Center, Rehovot and Hebrew University, Jerusalem, Israel.

${ }^{2}$ Department of Family Medicine, Clalit Health Service, Israel.
} 
vide care and guidance in the critical period shortly after birth may prove to have significant and long-lasting effects.

In 1999-2000, the authors conducted a prospective study of the duration of breastfeeding and factors influencing its cessation in a cohort of Israeli mother-infant pairs (cohort 1$).^{3}$ In this study, the proportion of mothers initiating breastfeeding in the nursery was found to be reassuringly high at $84 \%$. However, the fall-off in breastfeeding rates over the first months was found to be high, suggesting that the fundamentals of breastfeeding knowledge and proficiency had not been widely acquired. Thus, only $25 \%$ were still breastfeeding at 6 months.

As a result of these findings, the authors explored various approaches to improving breastfeeding rates. In particular, it was their contention that the hours and days of the initiation of breastfeeding may constitute a critical period whose influence may extend to include the overall duration of breastfeeding. If so, an intensive intervention aimed at improving the success of breastfeeding initiation may be expected to improve long-term breastfeeding success.

This article reports on the effect of the administration of a course on breastfeeding guidance to nurses, midwives, and physicians in a hospital delivery room and newborn nursery on the duration of breastfeeding in an urban Israeli population.

\section{METHODS}

The study was conducted in the departments of neonatology and obstetrics at Kaplan Medical Center, Rehovot, Israel and received local ethics committee approval. The intervention was a 32-hour course of theoretical and practical study in breastfeeding that was developed together with the central administration of Clalit Health Services and the Israel Ministry of Health. During 2001-2002, this course was administered to all of the staff (nurses and physicians) in the newborn nursery and the postnatal wards and the midwives in the delivery room. The course faculty included neonatologists, an obstetrician, a dietician, a lactation consultant, and nurses. The topics of study included the health advantages of breast milk, breastfeeding in the delivery room, physiology of lactation, infectious diseases and contraindications to breastfeeding, practical lactation counseling and troubleshooting, maternal nutritional influences, social and economic aspects, human milk for premature infants, jaundice, medications and human milk, and the WHO ten steps to successful breastfeeding.

At an observational level, the main changes in practice resulting from this intervention were increased breastfeeding in the delivery room, markedly increased time and quality of effort devoted to individual breastfeeding assistance with mothers and increased hesitation before suggesting formula supplementation, even in the presence of significant postnatal weight loss. In addition, all forms of advertising or marketing of infant formulas (either direct or subtle) were removed from the environment of the obstetric and neonatal departments. In the 1999 cohort, rooming-in was employed only on rare occasions at the request of the mother. By 2003, rooming-in was widely promoted and $24 \%$ of mothers elected for this form of care. Gradually, initiatives for improving breastfeeding guidance began to be developed by the staff themselves.

In order to study and quantify the effects of this intervention, we conducted a follow-up study of the duration of breastfeeding in a similar cohort of mothers and infants during 2003-2004 (cohort 2).

\section{Study cohorts}

In order to compare both groups, the follow up study was conducted in exactly the same way as the preliminary study. Inclusion criteria were the same for both cohorts and included mothers with healthy infants born at term (37-42 weeks) during the study period. Mother-infant dyads were included on days when the investigators (MS, YC) were available. Exclusion criteria included prematurity and admission to the intensive care nursery for conditions requiring oxygen therapy, antibiotics, or intravenous fluids. Routine neonatal problems such as jaundice did not constitute criteria for exclusion.

Questionnaires were distributed to mothers 1 to 2 days after delivery and collected by the 
study physician (MS, YC) later the same day. The questionnaire was written in Hebrew and, in those few cases of language difficulties with mothers of Ethiopian or Russian origin, a translator assisted with filling in the form. The questionnaire included information on the mother's social, educational, family, employment, and medical history, her past breastfeeding experience and plans for feeding this infant, mode of delivery and use of epidural or other analgesia, availability of breastfeeding guidance, and current mode of feeding the infant.

The duration of breastfeeding, factors influencing its discontinuation and availability of breastfeeding guidance were assessed by sequential telephone calls during the first 12 months after birth. The phone calls included data collection and the investigators did not offer any additional guidance. Breastfeeding was defined as complete if breast milk was the sole source of milk that the infant received, whether the infant was receiving other forms of nutrition or not.

Statistical analysis was conducted using SPSS for Windows, Version 12. Univariate comparisons between the cohorts employed student's $t$-tests for continuous variables and chi-square analyses for categorical variables. Multivariate logistic regression analyses were employed in order to adjust for potential confounding variables.

\section{RESULTS}

The two study cohorts were similar in baseline characteristics, such as age, parity, and ed- ucational and religious background (Table 1). There was a small but significant increase in the proportion of mothers (not including primiparas) who had breastfed previous children (cohort 1, 85\%; cohort 2, 91\%; $p=0.032$ ). There was a nonsignificant trend toward longer duration of breastfeeding of the previous infant that was $3.5 \pm 4.9$ months in 1999 and $4.1 \pm 5.7$ months in $2003(p=0.13)$. However, on arrival at the delivery room, the proportion of mothers who planned to breastfeed the current infant was the same in both cohorts $(88 \%)$.

The rate of delivery by Cesarean section was unchanged at $20 \%$. However, the proportion of mothers receiving epidural anesthesia during labor rose markedly from $38 \%$ in cohort 1 to $67 \%$ in cohort $2(p=0.0001)$. This reflects a change in departmental policy regarding 24hour availability of this service.

As shown in Table 2, the pattern of breastfeeding changed strikingly between the two cohorts. The proportion of mothers who breastfed in the delivery room rose from $3 \%$ to $37 \%$ $(p=0.0001)$. The proportion of mothers who expressed satisfaction with the breastfeeding guidance and support they received during their hospital stay rose from $43 \%$ in 1999 to $79 \%$ in $2003(p=0.0001)$. The mean duration of complete breastfeeding rose from $3.7 \pm 3.7$ months in 1999 to $5.6 \pm 4.3$ months in 2003 ( $p=$ $0.0001)$. The proportion of infants who were completely breastfeeding at each month of the first year of life in both cohorts is shown in Figure 1. In particular, the proportion of infants who were completely breastfeeding at 6 months of age rose from $26 \%$ in cohort 1 to $47 \%$ in cohort 2.

Table 1. Baseline Data in Both Study Cohorts

\begin{tabular}{llccc}
\hline Variable & & 1999 & 2003 & $\mathrm{p}$ \\
\hline $\mathrm{N}$ & & 471 & 364 & \\
Maternal age & & $29.9 \pm 5.3$ & $30.6 \pm 5.1$ & 0.099 \\
Previous children & $1.6 \pm 1.6$ & $1.4 \pm 1.4$ & 0.034 \\
Maternal education & Junior & $6 \%$ & $5 \%$ & 0.48 \\
& High school & $55 \%$ & $36 \%$ & \\
Religious identity & College + & $39 \%$ & $35 \%$ & \\
& Secular & $35 \%$ & $38 \%$ & \\
& Traditional & $37 \%$ & $18 \%$ & \\
Cesarean section & Orthodox & $20 \%$ & $9 \%$ & 0.70 \\
Epidural anesthesia & Ultra-orthodox & $70 \%$ & $20 \%$ & 0.6 \\
\hline
\end{tabular}


Table 2. Patterns of Breastfeeding

\begin{tabular}{lcccc}
\hline Variable & 1999 & 2003 & $\mathrm{p}$ & ERR (95\% CI) \\
\hline $\begin{array}{l}\text { Breastfed in past } \\
\quad \text { (excluding primiparas) }\end{array}$ & $85 \%$ & $91 \%$ & 0.032 & $1.07(1.02,1.13)$ \\
$\begin{array}{l}\text { Breastfed in past } \\
\quad \text { (excluding primiparas, }\end{array}$ & $3.5 \pm 4.9$ & $4.1 \pm 5.7$ & 0.13 & $\mathrm{n} / \mathrm{a}$ \\
$\quad$ duration, months) & & & \\
Plan to breastfeed & $88 \%$ & $88 \%$ & 0.91 & $1.00(0.95,1.05)$ \\
Breastfed in DR & $3 \%$ & $37 \%$ & 0.0001 & $12.5(7.3,21.3)$ \\
Breastfed in nursery & $84 \%$ & $93 \%$ & 0.0001 & $1.11(1.06,1.16)$ \\
Breastfeeding duration & $3.7 \pm 3.7$ & $5.6 \pm 4.3$ & 0.0001 & $\mathrm{~N} / \mathrm{A}$ \\
$\quad$ (months) & $26 \%$ & $47 \%$ & 0.0001 & $1.81(1.50,2.19)$ \\
Breastfed at 6 months & $43 \%$ & $79 \%$ & 0.0001 & $1.84(1.63,2.06)$ \\
Satisfied with lactation & & & \\
$\quad$ counseling in nursery & & & & \\
\hline
\end{tabular}

The reasons stated by mothers for stopping breastfeeding changed a little between the two cohorts (Table 3). The proportion of mothers citing inadequate milk supply, maternal health problems or inadequate guidance fell slightly. By comparison, mothers citing infant health problems, such as inadequate weight gain and return to work rose between the two cohorts.

Multivariate linear regression analysis, including factors that were significant on univariate analysis, found the study cohort to be the most powerful independent predictor of duration of complete breastfeeding $(p=0.0001)$. Other significant independent predictors were previous breastfeeding experience $(p=0.0001)$ and breastfeeding the current infant in the delivery room $(p=0.029)$.

As mentioned, rooming-in was only available in cohort 2 in 2003. Comparison of the duration of complete breastfeeding in infants whose mothers had used partial or complete rooming-in is shown in Table 4. No significant difference in the duration of breastfeeding was found between the groups of infants based on rooming-in.

\section{DISCUSSION}

This study demonstrated a marked increase in breastfeeding between 1999 and 2003 in the population served by a local general hospital. This change was temporarily associated with implementation of an intensive course of breastfeeding guidance that was provided to the obstetric and neonatal staff.
It is tempting to conclude from these data that the breastfeeding course directly resulted in part or all of the rise in breastfeeding rates in the population. However, although highly suggestive, the historical comparative design of this study precludes such a categoric conclusion. With this caveat, consider the following findings that appear to support the contention that this intervention contributed significantly to the rise in breastfeeding:

1. As there has been a rise in awareness among the general population regarding the importance of breastfeeding, the authors attempted to isolate the effect of the intervention by studying the intentions of mothers on arrival at the delivery room. No difference was detected between the two cohorts, suggesting that there has not been a signif-

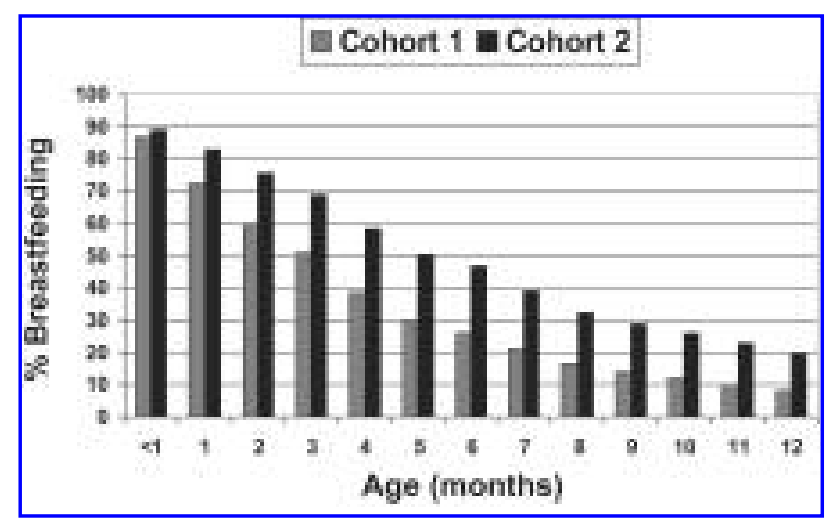

FIG. 1. Proportion of mothers who were breastfeeding at each month of age during the first year of life. All those who continued breastfeeding after age 12 months were categorized as 12 months. 
TABle 3. Reasons for DiscontinUing BREASTFEeding

\begin{tabular}{lrrr}
\hline Variable & 1999 & 2003 & $\mathrm{p}$ \\
\hline $\mathrm{N}$ & 471 & 364 & \\
Inadequate milk supply & $35 \%$ & $28 \%$ & \\
Return to work & $16 \%$ & $20 \%$ & \\
Maternal health reason & $15 \%$ & $10 \%$ & 0.0001 \\
Infant health reason & $5 \%$ & $21 \%$ & \\
Others (including insufficient & $29 \%$ & $22 \%$ & \\
$\quad$ support or guidance) & & & \\
\hline
\end{tabular}

icant change in attitudes toward breastfeeding in this population.

2. One of the best-known predictors of successful breastfeeding is breastfeeding shortly after birth, even in the delivery room. As a result of the intervention in this study, a marked change in the opinions of the staff was noted about the importance of encouraging the mothers to breastfeed in the delivery room. Accordingly, the proportion of mothers who breastfed in the delivery room rose markedly.

3. There was an almost doubling of the proportion of mothers who expressed satisfaction with the breastfeeding guidance they received during their stay in the hospital.

4. The first days after birth constitute a "critical period" in infant development and input at this stage has a profound influence on subsequent behavior. Indeed, a recent study of the reasons for stopping and the timing of critical periods in breastfeeding concluded that there is a "need to provide extensive breastfeeding support after delivery." 4

However, despite these contentions, it may be that interventions at community level influenced the rates of breastfeeding in the period between the two study cohorts. The Israel Ministry of Health and Health Maintenance Organizations conducted breastfeeding guidance courses for nurses in Mother-Infant Welfare Clinics and breastfeeding has been a major part of health promotion campaigns in recent years. Unfortunately, no data are available to quantify possible changes in national breastfeeding rates during this period. However, one finding addresses the limited efficacy of community interventions. In a 2004 study of 593 Israeli moth- ers, only $37 \%$ reported receiving breastfeeding guidance after discharge from the hospital. ${ }^{5}$ In addition, the rates and duration of breastfeeding in this cohort 1 in this study is similar to that reported in other recent Israeli studies. ${ }^{6-9}$

In this study, the intervention was aimed at health care providers. By comparison, a number of studies have focused on health education interventions provided to pregnant women and mothers. In the Cochrane review of the effect of these interventions on the rate of initiation of breastfeeding, seven randomized trials were identified. ${ }^{2}$ Although these studies demonstrated a significant effect on initiation rates, they were mostly performed in the United States in low-income groups with low overall breastfeeding rates. Thus, it is not clear whether such interventions would be effective in settings such as the authors' with approximately $90 \%$ initiation rates. Also, such interventions are highly labor-intensive and costly. Thus, it is gratifying to note that the indirect intervention chosen in this study appears to have made a major contribution despite the relatively small direct investment.

This study was performed in the setting of a mostly middle-class urban population with relatively high breastfeeding rates. The WHO Baby Friendly Hospital Initiative is currently practiced in 19,250 hospitals worldwide, of which fewer than 500 are in industrialized countries. The intervention in this study was tapered to the local population and included certain, but not all of the components of the WHO initiative. In particular, despite much encouragement, approximately $70 \%$ to $80 \%$ of the mothers continue to refuse rooming-in, prefer to breastfeed only at specified hours and provide artificial teats (3 of 10 steps). The finding of highly significant improvement in breastfeeding rates despite these limitations is interesting, suggesting that perhaps the influence of rooming-in varies among different population groups.

TAble 4. RoOMING-IN AND BREASTFEeding

\begin{tabular}{lc}
\hline Rooming-in & Breastfeeding (months) \\
\hline None & $5.4 \pm 4.4$ \\
Daytime only & $5.9 \pm 4$ \\
Day + night & $5.9 \pm 4.7$ \\
\hline
\end{tabular}


In summary, training hospital nursery staff in breastfeeding guidance is a potential, costeffective intervention even in settings with relatively high rates of breastfeeding.

\section{REFERENCES}

1. World Health Organization, Statement of the 54th Health Assembly, 2002.

2. Dyson L, McCormick F, Renfrew MJ. Interventions for promoting the initiation of breastfeeding. Cochrane Reviews 2005;CD001688:2

3. Shani M, Shinwell ES. Breastfeeding characteristics and reasons to stop breastfeeding. Harefuah 2003; 142:426-428.

4. Ahluwalia IB, Morrow B, Hsia J. Why do women stop breastfeeding? Findings from the pregnancy risk assessment and monitoring system. Pediatrics 2005;116: 1408-1412.

5. Clalit Health Services. Survey of Breastfeeding in the Community, 2004.

6. Clalit Health Services. Breastfeeding Survey, 2002.
7. Berger-Achituv S, Shohat T, Garty B. Breastfeeding patterns in Central Israel. IMAJ 2005;7:515-519.

8. Zur M, Shmuel BS, Bilenko N, et al. Breastfeeding patterns according to ethnic origin in two Well Child Care clinics in Beersheva and Ofakim. Harefuah 2004; 143:475-478.

9. Chertok IR, Shoham-Vardi I, Hallak M. Four-month breastfeeding duration in post-cesarean women of different cultures in the Israeli Negec. I Perinatol Neonatal Nurs 2004;18:145-160.

10. Philipp BL, Radford A. Baby-friendly: Snappy slogan or standard of care? Arch Dis Child Fetal Neonatal Ed 2006;91:F145-149.

Address reprint requests to: Eric S. Shinwell, M.D. Department of Neonatology Kaplan Medical Center Hebrew University P.O. Box 1 Rehovot 76100 Israel

E-mail: eric_s@clalit.org.il 